\title{
Semantic Web Research Trends and Directions
}

\author{
Jennifer Golbeck ${ }^{1}$, Bernardo Cuenca Grau, Christian Halaschek-Wiener, \\ Aditya Kalyanpur, Bijan Parsia, Andrew Schain, \\ Evren Sirin, and James Hendler \\ MINDSWAP, University of Maryland, College Park, MD 20742, USA \\ bernardo.cuenca@uv.es, bparsia@isr.umd.edu, andrew@schain.org, \\ \{golbeck, halasche, aditya, evren, hendler\}@cs.umd.edu \\ http://www . mindswap.org
}

\begin{abstract}
The Semantic Web is not a single technology, but rather a collection of technologies designed to work together. As a result, research on the Semantic Web intends both to advance individual technologies as well as to integrate them and take advantage of the result. In this paper we present new work on many layers of the Semantic Web, including content generation, web services, e-connections, and trust.
\end{abstract}

\section{Introduction}

Defining the Semantic Web is a difficult task. It is the next generation of the web. It is a set of languages and standards. It has a strong logic and reasoning component. Web services, web portals, markup tools, and applications are all components. As a result, there are many interesting, intertwined research areas.

In this paper, we address several emerging trends of research in the Semantic Web space. We begin by presenting two tools for creating content on the Semantic Web: SWOOP, an ontology browser and editor, and PhotoStuff, an image annotation tool that integrates with a web portal. We follow with descriptions of E-Connections, a logical formalism for combining ontologies, and of work in web services. Finally, we describe a project using social trust on the semantic web that builds upon the previous work to create end user applications that benefit from the semantic foundation.

\section{Swoop - Web Ontology Editor}

Swoop is a hypermedia-inspired Ontology Browser and Editor based on OWL, the first standardized Web-oriented ontology language. Swoop takes the standard Web browser as the UI paradigm, believing that URIs are central to the understanding and construction of OWL Ontologies.

All design decisions are in keeping with the OWL nature and specifications. Thus, multiple ontologies are supported easily, various OWL presentation syntax are used to render ontologies, open-world semantics are assumed while editing and OWL reasoners can be integrated for consistency checking. A key point in our work is that the hypermedia basis of the UI is exposed in virtually every 
aspect of ontology engineering — easy navigation of OWL entities, comparing and editing related entities, search and cross referencing, multimedia support for annotation, etc. - thus allowing ontology developers to think of OWL as just another Web format, and thereby take advantage of its Web-based features.

\subsection{Summary of Features}

Swoop functionality is characterized by the following basic features (for an elaborate discussion of the features see [4]).

- Multiple Ontology Browsing and Editing

- Renderer Plugins for OWL Presentation Syntaxes

- Reasoner plugins in Swoop

- Semantic Search

- Ontology Versioning

Additionally, Swoop has the following advanced features, which represent work in progress:

- Resource Holder (Comparator/Mapping utility)

- Automatic Partitioning of OWL Ontologies (using E-Connections): Swoop has a provision for automatically partitioning OWL Ontologies by transforming them into an E-connection. For more details on the theory and significance of E-connections, their use in the context of OWL Ontologies see section 4.

- Ontology Debugging and Repair

- Annotea Client in Swoop: Collaborative Annotations and Change Sets.

\section{PhotoStuff Semantic Image Annotation Tool}

PhotoStuff is a platform-independent image annotation tool that allows users to annotate regions of an image with respect to concepts in any ontology specified in RDFS or OWL. It provides the functionality to import images (and their embedded metadata), ontologies, instance-bases, perform markup, and export the resulting annotations to disk or a Semantic Web portal.

\subsection{Overview}

PhotoStuff is designed to load multiple ontologies at once, enabling a user to markup images with concepts distributed across any of the loaded ontologies.

The ontologies are visualized in both a class tree and list (depicted below in Figure 1 in the far left pane of the tool). User can load images (from the Web and/or local disk) in PhotoStuff. The terms listed in both the tree and list can be dragged into any region, or into the image itself, creating a new instance of the selected class. An instance creation form is dynamically generated from the properties of the selected class (range restrictions are imposed). Especially valuable, existing instances can be loaded from any URI on the Web. Using these preloaded instances, depictions can reference existing instances. 


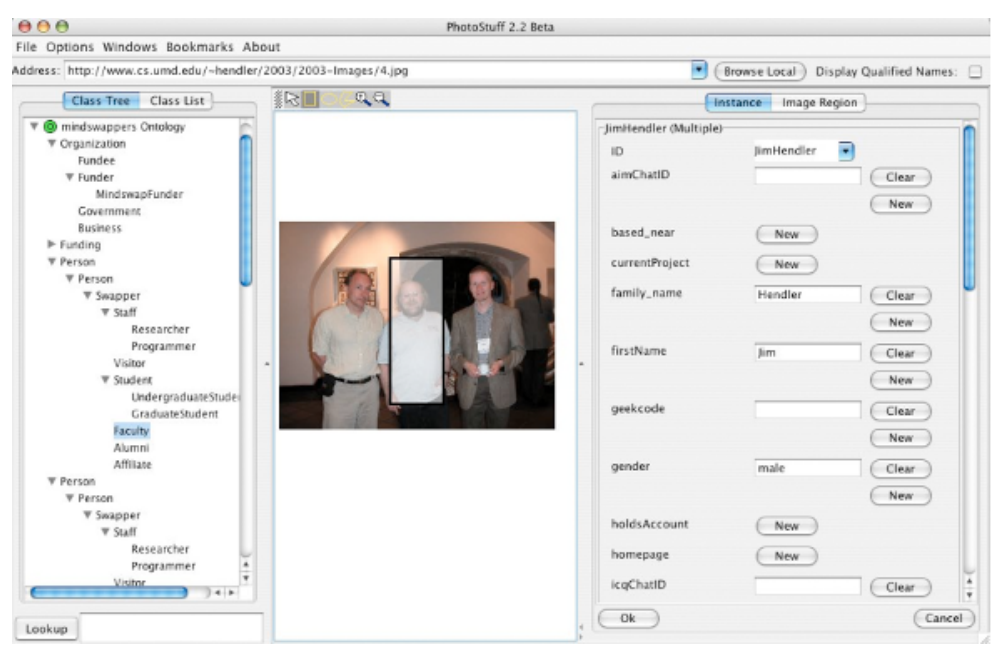

Fig. 1. PhotoStuff Screenshot

PhotoStuff maintains a loose coupling with a Semantic Web portal There are three ways in which PhotoStuff interacts with the portal, namely retrieving all instances that have been submitted to the portal, submitting generated $\mathrm{RDF} / \mathrm{XML}$, and uploading local images so they can be referenced by a URI (thus allowing them to be referenced using RDF/XML).

\subsection{Image Metadata Browsing and Searching}

After metadata of annotated images is submitted to the Semantic Web portal, semantics based image browsing and searching is provided. The portal uses information from the various ontologies image content is annotated against to guide the display of and interaction with metadata All instances that are depicted within an image are presented. As noted earlier, the underlying class of each instance drives the actual order of the depictions, thus providing a high level view of all the metadata of images that have been annotated using PhotoStuff.

When an instance is selected, the user is presented with all images in which the instance is depicted (illustrated in Figure 2). All of the metadata regarding that instance is presented to the user as well (currently in tabular form). We note here that this links the images with pre-existing metadata maintained in the portal.

In Figure 2, it can be seen that specific regions are highlighted. This is accomplished using an SVG outline of the region drawn on the various images (this data is embedded in RDF/XML as well). By selecting an image region, the various co-regions of the selected image region are displayed (shown in Figure 2). This allows browsing of the metadata associated with the various regions depicted in the image. Lastly, the portal provides support for searching image metadata. Images are searchable at the instance and class level. 


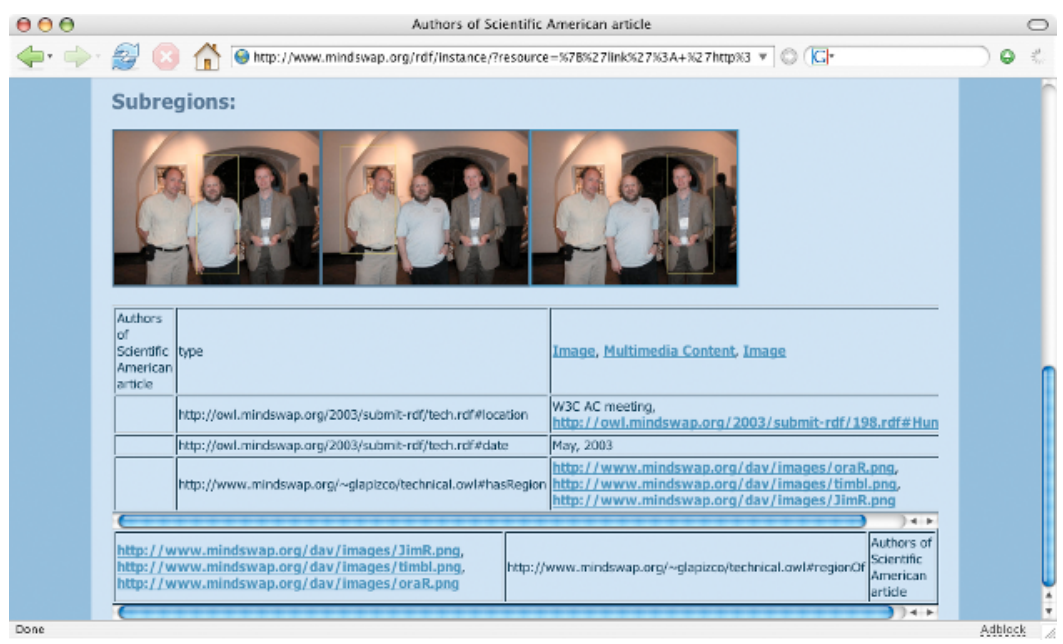

Fig. 2. Instance Descriptions and Image Co-Region Browsing

\section{E-Connections of Web Ontologies}

An E-Connection is a knowledge representation (KR) formalism defined as a combination of other logical formalisms. The component logics that can be used in an E-Connection include Description Logics (and hence OWL-DL), some temporal and spatial logics, Modal and Epistemic logics. E-Connections were originally introduced as a way to go beyond the expressivity of each of the component logics, while preserving the decidability of the reasoning services. Obviously, different component logics will give rise to different combined languages, with different expressivity and computational properties.

In a Semantic Web context, E-Connections allow the user to combine OWLDL ontologies. A combination of OWL-DL ontologies is called a combined knowledge base. A combined knowledge base is a set of "connected ontologies. These connected ontologies are basically OWL-DL ontologies extended with the ability to define and use link properties.

In OWL-DL, object properties are used to relate individuals within a given ontology, while datatype properties relate individuals to data values. In a combined knowledge base, link properties are used to relate individuals belonging to different ontologies in the combination.

A link property can be used to define classes in a certain ontology in terms of other classes corresponding to different ontologies in the combination. For example, a "Graduate Student" in an ontology about "people" could be defined as a student who is enrolled in at least one graduate course, by using the class "Student" in the people ontology and a someValuesFrom restriction on the link property "enrolledIn" with value "GraduateCourse": a class in a different ontology dealing with the domain of "academic courses". 
Link properties are logically interpreted as binary relations, where the first element belongs to the "source" ontology and the second to the "target ontology" of the link property. Conceptually, a link property will be defined and used in its "source" ontology. For example, the link property "enrolledIn" would be defined as a link property in the "people" ontology with target ontology "academic courses".

From the modeling perspective, each of the component ontologies in an EConnection is modeling a different application domain, while the E-Connection itself is modelling the union of all these domains. For example, an E-Connection could be used to model all the relevant information referred to a certain university, and each of its component ontologies could model, respectively, the domain of people involved in the university, the domain of schools and departments, the domain of courses, etc.

\subsection{Applications}

Integration of OWL Ontologies. E-Connections can be used as a suitable representation for combining and integrating OWL-DL ontologies on the Semantic Web. For example, suppose a set of ontologies that have been independently developed and are now required to interoperate within an application. In order to provide support for integrating Web ontologies, OWL defines the owl:imports construct, which allows to include by reference in a knowledge base the axioms contained in another ontology, published somewhere on the Web and identified by a global name (a URI). However, the only way that the owl:imports construct provides for using concepts from a different ontology is to bring into the original ontology all the axioms of the imported one. This keeps the ontologies in separate files, providing some syntactic modularity, but not a logical modularity, as in the case of E-Connections.

Analysis and Decomposition of OWL Ontologies. E-Connections have also proved useful for analyzing the structure of knowledge bases and, in particular, to discover relevant sub-parts of ontologies, commonly called modules. For example, suppose one wants to annotate a word in a document using a class from an ontology, published elsewhere on the Web. It would be natural for a Semantic Web application to retrieve only the minimal set of axioms that "capture" the meaning of that specific class in the remote ontology.

In general, the ability to identify relevant sub-parts of ontologies is important for virtually every Semantic Web application. For example, in ontology engineering, achieving some sort of "modularity" is a key requirement to facilitate collaborative, large scale, long term development. Modularity worthy of the name should provide benefits in the following aspects: processability of the ontology by applications, evolvability and maintenance, knowledge reuse and understandability for humans.

E-Connections provide a sensible way for identifying modules within an ontology. The main idea of the method is to transform the original ontology into an E-Connection with the largest possible number of connected knowledge bases 
such that it preserves the semantics of the original ontology in a very specific way. The obtained connected ontologies are used to generate, for each entity, a subset of the original ontology that encapsulates the entity, i.e., that preserves a certain set of crucial entailments related to the entity. The key advantage of relying on E-Connections for such a task is that modules are obtained in a completely automatic way, without relying on any heuristics or user intervention.

Tool Support. We have provided support for E-Connection in both an OWL ontology editor, SWOOP, and an OWL reasoner, Pellet. Our aim has been to build an E-Connection aware infrastructure that people with a large commitment to OWL will find understandable and useful. We have been mostly concerned with reusing as much of our current tool support for OWL as possible. Our experience in implementing OWL tools has taught us that implementing a SemanticWeb Knowledge Representation formalism is different from implementing the very same formalism outside the Semantic Web framework. In particular, any new formalism for the Semantic Web needs to be compatible with the existing standards, such as RDF and OWL. For such a purpose, we have extended the syntax and semantics of the OWL-DL recommendation. Thus, we have explored both the issues related to the implementation of a new KR formalism, E-Connections, and those concerning its integration in a Semantic Web context.

\section{Web Services}

In this section, we describe various different projects and applications we developed for Web Services. Our work on Web Services concentrates on three core tasks: matchmaking, composition, and execution; always focusing on the end-toend experience. To enable the automation of such tasks, we needed expressive descriptions of Web Services. For this reason, we have been involved with the development of the OWL-S language. The OWL-S is a collection of ontologies written in OWL to describe Web Services. Currently it is the most mature and probably the most widely deployed comprehensive Semantic Web Service technology.

The OWL-S descriptions are invaluable for many web service tasks. However, the intended semantics of OWL-S service descriptions is not expressed (or expressable, often) in OWL. Furthermore, working with OWL-S descriptions at the RDF or even the OWL level is quite difficult and tedious as they tend to be at the wrong level of abstraction. For this reason, we have developed the Mindswap OWL-S API [6], a Java API developed for parsing, validating, manipulating, executing, matching, and in general reasoning over OWL-S descriptions. OWL-S API provides a programmatic interface that has been designed closely to match the definitions in the OWL-S ontology. This interface helps developers build applications using OWL-S without having to deal with all the details of the syntax. The API provides an execution engine to invoke atomic services as well as composite processes that are built using the OWL-S control constructs.

Our work on Semantic Web Services focuses on the automated composition of services using Hierarchical Task Network (HTN) planning formalism [2]. Instead 
of looking at each service in isolation, we focus on the descriptions of workflow templates. Workflow templates are used for various different tasks such as encoding business rules in a B2B application, specifying domain knowledge in a scientific Grid application, and defining preferences for users that interact with Web Services. A template describes the general outline of how to achieve a certain task and the planners job is to find which of these possible execution paths will be successful in the current state of the world with the current requirements and preferences.

We have developed a mapping [7] from OWL-S to the HTN formalism as implemented in the SHOP2 planner [5]. Using this translation, we implemented a system that plans over sets of OWL-S descriptions using SHOP2 and executes the resulting plans over the Web. The planning system is also capable of executing information-providing Web Services during the planning process to decide which world-altering services to use in the plan. It is possible to completely automate the information gathering process by inspecting the preconditions of a service and finding the relevant information-providing services during planning [8].

The problem of using a planner for composing OWL-S services is the limited reasoning capabilities provided by the planner. The typical logic for expressing preconditions and effects in a planning system has a radically different expressiveness then RDF and OWL do. In order to evaluate such formulas, the planners must understand the semantics of OWL. We have integrated the SHOP2 planner with our OWL-DL reasoner Pellet to overcome this problem [9]. In this integrated system, the precondition evaluation is done by the OWL reasoner against the local and remote OWL ontologies. Such integration poses some efficiency challenges because the planner constantly simulates the effects of services resulting in modifications to its internal OWL KB and continues to query the $\mathrm{KB}$ as it continues the planning process. We have developed several query optimization methods to increase the performance of the system.

Most recently, we are looking at how to handle more expressive workflow templates where HTN task selection is extended to incorporate OWL-S based matchmaking [10]. We aim to extend the OWL-S language to describe the abstract processes using profile hierarchies and complex OWL concept descriptions. This extension will allow a clearer way to define soft and hard preferences in a template along with a prioritization of these preferences. Consequently, we can have more elaborate ranking mechanisms for choosing out of the possible options during planning. We are currently working on the implementation of this extended HTN formalism (HTN-DL) [10].

\section{Trust: Computations and Applications}

One of the ultimate goals of the Semantic Web is to produce a so-called "Web of Trust". Much work in this space has been made in the spheres of security, authentication, and privacy. However, the social component of trust is one that is both important and ideally suited for the Semantic Web. When the Semantic Web-based social networks are augmented with trust information, it is possible to make computations over the values, and integrate the results into applications. 


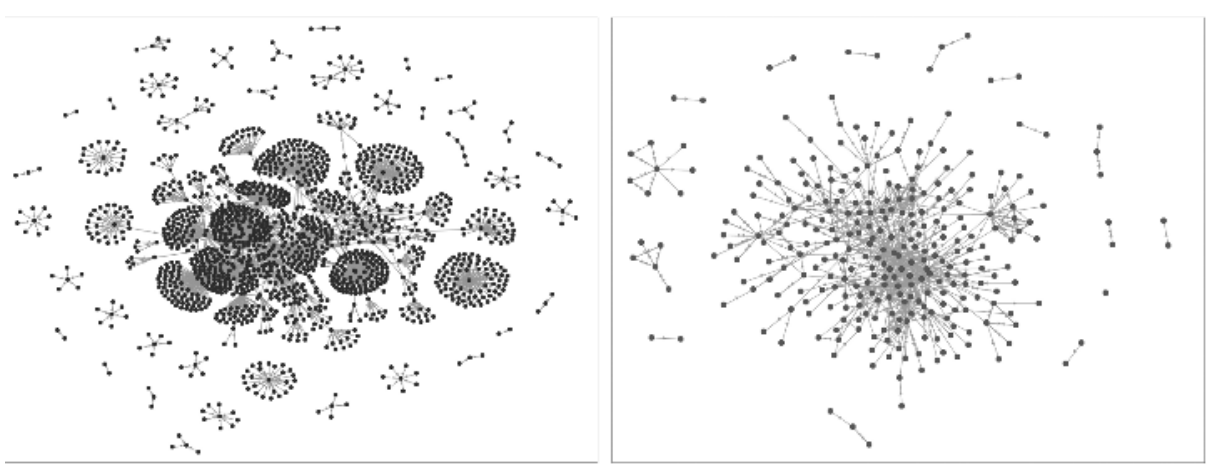

Fig. 3. Visualizations of the Trust Project's network (left) and the FilmTrust network (right)

\subsection{Adding Trust to FOAF}

In the FOAF vocabulary, the only mechanism for describing social connections between people is the foaf:knows property which indicates one person knows another. The FOAF Trust Module extends the FOAF vocabulary by providing a mechanism for describing the trust relationships. It allows people to rate the trustworthiness of another person on a scale from 1 to 10 where 1 is low trust and 10 is a high trust. This scale is intuitive for human users, but our algorithms can be applied to any scale of values.

The Trust Project ${ }^{1}$ regularly spiders the Semantic Web for trust files, and maintains a network with over 2,000 people. The ontology is also used by the FilmTrust social network [3] with over 400 members. These networks can be seen in Figure 3, and are used as testbeds for our algorithms for computing trust.

\subsection{Computing with Trust}

If one person, the source, wants to know how much to trust another person, the sink, how can that information be obtained? Clearly, if the source knows the sink, the solution is simple. However, if the two do not know one another, the trust values within the social network can be used to compute a recommendation to the source regarding the trustworthiness of the sink.

Because social trust is an inherently personal concept, a computed trust value must also be personal. Thus, we do not compute a global measure of how trustworthy the sink is; rather, we use the source's perspective on the network to find paths of trust that are in turn used for making the trust computation.

TidalTrust is an algorithm for computing trust over a range of values, such as those provided by the FOAF Trust Module. It is a simple recursive algorithm: the source asks each of its neighbors for their trust rating of the sink. The source then computes a weighted average of these values, giving more weight to neighbors with higher trust ratings, and less weight to neighbors with lower

\footnotetext{
${ }^{1}$ see http://trust.mindswap.org
} 
trust ratings. If a neighbor has a direct trust rating of the sink, it returns that value; otherwise, the sink repeats the algorithm for its neighbors, and returns the weighted average that it computes. Because this algorithm is essentially a modified Breadth First Search, it runs in linear time with respect to the site of the network. More detail on the algorithm can be found at [3].

Previous work[3] has shown that the results returned by TidalTrust when it is run on both the Trust Project network and the FilmTrust network can be expected to be relatively accurate. The error varies from network to network, but is usually within $10 \%$.

\subsection{Applying Trust}

These trust computations are made generally, with networks on the Semantic Web. As such, they can be integrated into a variety of applications.

There are two major projects we have undertaken to illustrate the benefit of trust ratings to the user. The first is FilmTrust, a website built on a semantic social network with movie ratings and reviews from users. On the websit, the user's movie ratings are combined with trust values to generate predictive movie ratings. We have shown that, in FilmTrust, the trust-based predictive ratings outperform other methods of generating these predictions in certain situations[3]. The second application is TrustMail, and email client that uses the trust rating of a message's sender as a score for the email. The trust ratings work as the complement to a spam filter by allowing users to identify potentially important messages by the trustworthiness of their sender.

Because trust and social networks are general and available publicly on the Semantic Web, there is great potential for any application to integrate this data, make computations with it, and use the results to improve the user experience.

\section{Conclusions}

In this paper, we have presented tools and research projects for creating Semantic Web content with SWOOP and PhotoStuff, combining ontologies with E-Connections, working with web services, and computing with trust in Semantic Web-based social networks. These topics illustrate both the breadth and depth of research topics on the Semantic Web, and serve as clear examples of trends in Semantic Web research.

\section{Acknowledgements}

This work, conducted at the Maryland Information and Network Dynamics Laboratory Semantic Web Agents Project, was funded by Fujitsu Laboratories of America - College Park, Lockheed Martin Advanced Technology Laboratory, NTT Corp., Kevric Corp., SAIC, the National Science Foundation, the National Geospatial-Intelligence Agency, DARPA, US Army Research Laboratory, NIST, and other DoD sources. 


\section{References}

1. Cuenca Grau, Bernardo and Bijan Parsia. From shoq(d) toward e-connections. In Proceedings of the 2004 International Workshop on Description Logics, (DL2004), (Poster)., 2004.

2. Erol, Kutluhan, James Hendler, and Dana Nau. HTN planning: Complexity and Expressivity In Proceedings of the 12th National Conference on Artificial Intelligence (AAAI94), Seattle, 1994.

3. Golbeck, Jennifer. Computing and Applying Trust in Web-Based Social Networks, Ph.D. Dissertation, University of Maryland, College Park, 2005.

4. Kalyanpur, Aditya, Bijan Parsia, and James Hendler. A tool for working with web ontologies. In In Proceedings of the International Journal on Semantic Web and Information Systems, Vol. 1, No. 1, Jan - March, 2005.

5. Nau, Dana, Tsz-Chiu Au, Okhtay Ilghami, Ugur Kuter, J. William Murdock, Dan Wu, Fusun Yaman. SHOP2: An HTN planning system. Journal of AI Research, 20:379404, 2003.

6. Sirin, Evren and Bijan Parsia. The OWL-S Java API. (Poster) In Proceedings of the Third International Semantic Web Conference (ISWC2004), Hiroshima, Japan, 2004 .

7. Sirin, Evren, Bijan Parsia, Dan Wu, James Hendler, and Dana Nau. HTN Planning for Web Service Composition using SHOP2. Journal of Web Semantics, 1(4):377396, 2004.

8. Kuter, Ugur, Evren Sirin, Dana Nau, Bijan Parsia, and James Hendler. Information Gathering During Planning for Web Service Composition. In Proceedings of the Third International Semantic Web Conference (ISWC2004), Hiroshima, Japan, 2004.

9. Sirin, Evren, and Bijan Parsia. Planning for Semantic Web Services. In Semantic Web Services Workshop at the Third International Semantic Web Conference (ISWC2004),

10. Sirin, Evren, Bijan Parsia, and James Hendler. Template-based Composition of Semantic Web Services. Submitted to AAAI Fall Symposium on Agents and the Semantic Web, 2005. 